\title{
FREE DIVISORS VERSUS STABILITY AND COINCIDENCE THRESHOLDS
}

\author{
GABRIEL STICLARU
}

\begin{abstract}
We show that there is an unexpected relation between free divisors and stability and coincidence thresholds for projective hypersurfaces.
\end{abstract}

\section{INTRODUCTION}

Let $S=\mathbb{C}\left[x_{0}, \ldots, x_{n}\right]$ be the graded ring of polynomials in $x_{0}, \ldots, x_{n}$ with complex coefficients and denote by $S_{r}$ the vector space of homogeneous polynomials in $S$ of degree $r$. For any polynomial $f \in S_{r}$ we define the Jacobian ideal $J_{f} \subset S$ as the ideal spanned by the partial derivatives $f_{0}, \ldots, f_{n}$ of $f$ with respect to $x_{0}, \ldots, x_{n}$. For $n=2$ we use $x, y, z$ instead of $x_{0}, x_{1}, x_{2}$ and $f_{x}, f_{y}, f_{z}$ instead of $f_{0}, f_{1}, f_{2}$.

The Hilbert-Poincaré series of a graded $S$-module $M$ of finite type is defined by

$$
H P(M)(t)=\sum_{k \geq 0} \operatorname{dim} M_{k} \cdot t^{k}
$$

and it is known, to be a rational function of the form

$$
H P(M)(t)=\frac{P(M)(t)}{(1-t)^{n+1}}=\frac{Q(M)(t)}{(1-t)^{d}} .
$$

For any polynomial $f \in S_{r}$ we define the corresponding graded Milnor (or Jacobian) algebra by

$$
M=M(f)=S / J_{f}
$$

Smooth hypersurfaces of degree $d$ have all the same Hilbert-Poincaré series, namely

$$
H P(M(f))(t)=\frac{\left(1-t^{d-1}\right)^{n+1}}{(1-t)^{n+1}}=\left(1+t+t^{2}+\ldots+t^{d-2}\right)^{n+1} .
$$

As soon as the hypersurface $V(f)$ acquires some singularities, the series $H P(M(f))$ is an infinite sum.

Let $\tau(V(f))=\sum_{j=1, p} \tau\left(V(f), a_{j}\right)$ be the global Tjurina number of the hypersurface $V(f)$. In particular, the Hilbert polynomial $H(M(f))$ is constant and this constant is $\tau(V(f))$.

For a hypersurface $D: f=0$ in $\mathbb{P}^{n}$ with isolated singularities we recall the following invariants, introduced in [4].

2010 Mathematics Subject Classification. 13D40, 14J70, 14Q10, 32S25.

Key words and phrases. projective hypersurfaces, singularities, Milnor algebra, Hilbert-Poincaré series, free divisor. 
Definition 1.1. (i) The coincidence threshold ct $(D)$ defined as

$$
\operatorname{ct}(D)=\max \left\{q: \operatorname{dim} M(f)_{k}=\operatorname{dim} M\left(f_{s}\right)_{k} \text { for all } k \leq q\right\},
$$

with $f_{s}$ a homogeneous polynomial in $S$ of degree $d=\operatorname{deg} f$ such that $D_{s}: f_{s}=0$ is a smooth hypersurface in $\mathbb{P}^{n}$.

(ii) The stability threshold st $(D)$ defined as

$$
s t(D)=\min \left\{q: \operatorname{dim} M(f)_{k}=\tau(D) \text { for all } k \geq q\right\}
$$

where $\tau(D)$ is the total Tjurina number of $D$, i.e. the sum of all the Tjurina numbers of the singularities of $D$.

We refer for the definiton of free divisors and their main properties to [8] and the extensive reference list there.

\section{MAIN RESULT}

The main result of this note is the following.

Theorem 2.1. Let $f \in S_{d}$ and set as usual $T=(n+1)(d-2)$. Assume that $\operatorname{st}(D)<\operatorname{ct}(D)$. Then the following hold.

(1) The degree $d$ is odd, $\operatorname{ct}(D)=(T+1) / 2$ and $\operatorname{st}(D)=(T-1) / 2$.

(2) If in addition $n=2$, then the curve $D: f=0$ is a free divisor.

To prove the first claim is fairly easy. Recall that the coefficients $c_{k}$ of the HilbertPoincaré series of $M(f)$ form a (strict) log-concave sequence by [9], symmetric with respect to the middle point $T / 2$, i.e. $c_{k}=c_{T-k}$. It follows that one has strict inequalities $c_{k-1}<c_{k}$ for all $k \leq T / 2$. This implies that $s t(D) \geq T / 2$ when $d$ is even and $\operatorname{st}(D) \geq(T-1) / 2$ when $d$ is even.

Suppose we are in the case $d$ is even. Then $\operatorname{st}(D)>T / 2$ implies $\operatorname{st}(D) \geq \operatorname{ct}(D)$ as beyond $T / 2$ the sequence $c_{k}$ is strictly decreasing. Hence one should have $s t(D)=$ $T / 2$, which implies $c t(D)=\operatorname{st}(D)$.

Suppose now that we are in the case $d$ is odd. Then again $\operatorname{st}(D)>(T-1) / 2$ implies $s t(D) \geq \operatorname{ct}(D)$ as beyond $T / 2$ the sequence $c_{k}$ is strictly decreasing. Hence one should have $\operatorname{st}(D)=(T-1) / 2$, which implies $\operatorname{ct}(D)=\operatorname{st}(D)+1=(T+1) / 2$.

The proof of the second claim is much more subtle.

Recall that $D$ is a free divisor if and only if the Jacobian ideal $J_{f}$ is a perfect ideal, see for instance the bottom of page 1 in [8]. Next, the Corollary 1.2 in [8] implies the following fact: if the Castelnuovo-Mumford regularity $\operatorname{reg}(M(f))$ satisfies

$$
\operatorname{reg}(M(f)) \leq(3 d-7) / 2,
$$

then $J_{f}$ is perfect and one has $\operatorname{reg}(M(f)) \leq(3 d-7) / 2$ with $d$ odd.

Using [2], Corollary 4, we know that

$$
\operatorname{reg}(M(f))=\max \left(T-\operatorname{ct}(D), \operatorname{sat}\left(J_{f}\right)-1\right),
$$


where for any homogeneous ideal $I$, we let $\widehat{I}$ be the associated saturated ideal and we consider the graded artinian $S$-module

$$
S D(I)=\frac{\widehat{I}}{I},
$$

called the saturation defect module of $I$ and the saturation threshold sat $(I)$ defined as

$$
\operatorname{sat}(I)=\min \left\{q: \operatorname{dim} I_{k}=\operatorname{dim} \widehat{I}_{k} \text { for all } k \geq q\right\} .
$$

Now, it follows from $(1)$ above that $T-\operatorname{ct}(D)=(T-1) / 2=(3 d-7) / 2$, so we need to control $\operatorname{sat}\left(J_{f}\right)$. Corollary 2 in [2] tells us that

$$
\operatorname{sat}\left(J_{f}\right) \leq \max (T-\operatorname{ct}(D), \operatorname{st}(D)) .
$$

and hence in our situation $\operatorname{sat}\left(J_{f}\right)-1 \leq(T+1) / 2-1=(T-1) / 2=(3 d-7) / 2$. Hence $D$ is a free divisor. This completes the proof of Theorem 2.1.

Remark 2.2. Note that in fact $D$ is a free divisor if and only if $J_{f}=\widehat{J}_{f}$, see top of page 6 in [8], and hence if and only if $\operatorname{sat}\left(J_{f}\right)=0$. In particular, for a free divisor one has

$$
\operatorname{reg}(M(f))=T-\operatorname{ct}(D)
$$

\section{EXAMPLES OF HYPERSURFACES}

We show here examples of hypersurfaces with $\operatorname{st}(D) \leq \operatorname{ct}(D)$

Example 3.1. (a triangle and a conic+tangent) The example $D: f=x y z=0$ has $\operatorname{sat}\left(J_{f}\right)=0,2=\operatorname{ct}(D)>\operatorname{st}(D)=1$. The conic plus tangent $D: f=x\left(x z+y^{2}\right)$ has the same invariants and Hilbert-Poincaré series $H P(M(f))(t)=1+3(t+\cdots$.

Example 3.2. (two free line arrangements in $\mathbb{P}^{2}$ ) Consider the line arrangement

$$
D\left(\mathcal{A}_{3}\right): f=\left(x^{2}-y^{2}\right)\left(y^{2}-z^{2}\right)\left(x^{2}-z^{2}\right)=0 .
$$

The Hilbert-Poincaré series is:

$$
H P(M(f))(t)=1+3 t+6 t^{2}+10 t^{3}+15 t^{4}+18 t^{5}+19\left(t^{6}+\cdots .\right.
$$

Then $\operatorname{ct}\left(D\left(\mathcal{A}_{3}\right)\right)=\operatorname{st}\left(D\left(\mathcal{A}_{3}\right)\right)=6$ and the curve $D\left(\mathcal{A}_{3}\right)$ is free, since one can verify by Singular the vanishing $\operatorname{sat}\left(J_{f}\right)=0$. Similarly, for the line arrangement

$$
D: g=x y z f=x y z\left(x^{2}-y^{2}\right)\left(y^{2}-z^{2}\right)\left(x^{2}-z^{2}\right)=0,
$$

one has $c t(D)=10<s t(D)=11$ and the Hilbert-Poincaré series is: $1+3 t+6 t^{2}+$ $10 t^{3}+15 t^{4}+21 t^{5}+28 t^{6}+36 t^{7}+42 t^{8}+46 t^{9}+48 t^{10}+49\left(t^{11}+\cdots\right.$. So this curve is free for the same reason as above.

Example 3.3. (a sequence of free divisors constructed in [8], Prop. 2.2.) Consider the sequence of free divisors $D_{d}: f=y^{d-1} z+x^{d}+x^{2} y^{d-2}=0$, for $d \geq 5$ Then, for $d=5$ we have $s t(D)=4<5=\operatorname{ct}(D)$, hence we are in the setting of Theorem 2.1 (ii). For $d=6$, one has $\operatorname{st}(D)=6=c t(D)$, while for $d>6$ one has $\operatorname{st}(D)>\operatorname{ct}(D)$. So Theorem 2.1 (2) has no converse. 
Example 3.4. (Simis' free irreducible sextic, see [7]) Consider the sextic $D: f=0$, where

$$
f=4\left(x^{2}+y^{2}+x z\right)^{3}-27\left(x^{2}+y^{2}\right)^{2} z^{2} .
$$

Then $D$ has three $E_{6}$ singularities and one node $A_{1}$, hence $\tau(D)=19$. Moreover one has $c t(D)=s t(D)=6$ since the Hilbert-Poincaré series of $M(f)$ can be computed using for instance Singular and we get

$$
H P(M(f))(t)=1+3 t+6 t^{2}+10 t^{3}+15 t^{4}+18 t^{5}+19\left(t^{6}+\cdots .\right.
$$

This is one of the very few irreducible free divisors in $\mathbb{P}^{2}$ known to this day.

Example 3.5. (a sequence of curves $C_{d}$ with $\operatorname{st}\left(C_{d}\right)=\operatorname{ct}\left(C_{d}\right)$.) Let $C_{d}: f_{d}=0$ with $f_{d}(x, y, z)=f=x^{2} y^{2} z^{d-4}+x^{5} z^{d-5}+y^{5} z^{d-5}+x^{d}+y^{d}$ for $d \geq 5$. Then, for any $d \geq 5$, the curve $C_{d}$ has a unique singularity at the point $(0: 0: 1)$ of type $T_{2,5,5}$ with $\tau=10$. Then a computation by Singular suggests that $\operatorname{ct}\left(D_{d}\right)=\operatorname{st}\left(D_{d}\right)=3 d-9$ (verified for $5 \leq d \leq 15$ ). Moreover all these divisors are not free since $\operatorname{sat}\left(J_{f}\right)$ is not zero (verified for $5 \leq d \leq 15$ ).

Example 3.6. (a nodal hypersurface with one node) If $D$ is a nodal hypersurface in $\mathbb{P}^{n}$ having exactly one node, it follows from Example 4.3 (i) in 5 that $\operatorname{st}(D)=$ $c t(D)=T$. For nodal curve of degree 5, $C: f=x^{4} y+x^{3} y^{2}+y^{5}+x y^{2} z^{2}+\left(x^{2}+\right.$ $\left.x y+y^{2}\right) z^{3}=0$ our invariants are $\operatorname{ct}(C)=s t(C)=9$ and the Hilbert-Poincaré series of $M(f)$ is

$$
H P(M(f))(t)=1+3 t+6 t^{2}+10 t^{3}+12 t^{4}+12 t^{5}+10 t^{6}+6 t^{7}+3 t^{8}+\left(t^{9}+\cdots .\right.
$$

\section{Conclusion}

For a hypersurface $D: f=0$ in $\mathbb{P}^{n}$ with isolated singularities, we compare the Hilbert-Poincare series of the corresponding graded Milnor algebra with the smooth case. The series coincides up to a point $\operatorname{ct}(D)$ and Choudary-Dimca Theorem [1] ensures that from an index $s t(D)$ we have stability.

The main result is Theorem 2.1, which establishes a relation between free divisors and stability $s t(D)$ and coincidence thresholds $c t(D)$.

In the vast majority of cases one has $c t(D) \leq s t(D)$, see for instance the examples given above for the case of equality. The opposite inequality $\operatorname{st}(D)<c t(D)$ may occur but it is extremely rare.

Recall that one of the main question in this theory is the construction of irreducible free divisor of arbitrarily high degree. Simis' celebrated example in degree $d=6$ is discussed in Example 3.4. The main conclusion of the given examples is that Theorem 2.1 (2) has no converse, even in the class of free line arrangements. Moreover, the examples show that there are a lot of free and not free divisors satisfying the equality $\operatorname{st}(D)=\operatorname{ct}(D)$.

All the computations for this paper were made by Singular package, see [3] and [6].

\section{Acknowledgment}

The author is grateful to A. Dimca for suggesting this problem. 


\section{REFERENCES}

[1] A. D. R. Choudary, A. Dimca, Koszul complexes and hypersurface singularities, Proc. Amer.

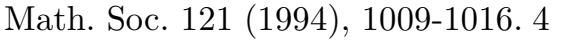

[2] A. Dimca, Syzygies of Jacobian ideals and defects of linear systems, Bull. Math. Soc. Sci. Math. Roumanie Tome 56(104) No. 2, 2013, 191- 203. 2, 2

[3] W. Decker, G.-M. Greuel, G. Pfister and H. Schönemann, Singular - A computer algebra system for polynomial computations. Available at http://www.singular.uni-kl.de. 4

[4] A. Dimca, G. Sticlaru, On the syzygies and Alexander polynomials of nodal hypersurfaces, Math. Nachr. 285, No.17-18, (2012), 2120-2128.1

[5] A. Dimca, G. Sticlaru, Koszul complexes and pole order filtrations, P Edinburgh Math Soc, accepted 2013. 3.6

[6] G.-M. Greuel, G. Pfister, A Singular Introduction to Commutative Algebra (with contributions by O. Bachmann, C. Lossen, and H. Schnemann). Springer-Verlag 2002 (second edition 2007). 4

[7] A. Simis: The depth of the jacobian ring of a polynomial in three variables. Proc. AMS 134 (2005), 1591-1598. 3.4

[8] A. Simis, S.O. Tohaneanu: Homology of homogeneous divisors. arXiv:1207.5862. 1, 2, 2.2, 3.3

[9] G. Sticlaru, Invariants and rigidity of projective hypersurfaces, arXiv: 1309.7356. 2

Faculty of Mathematics and Informatics, Ovidius University, Constanta, Romania

E-mail address: gabrielsticlaru@yahoo.com 